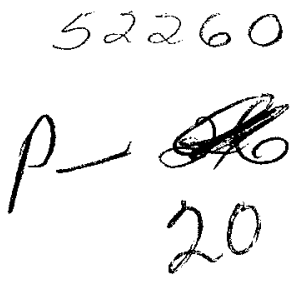

SEMI-ANNUAL REPORT TO NASA LANGLEY UNDER GRANT NAG-1-1119

\author{
by \\ Stewart A L Glegg, \\ Department of Ocean Engineering, \\ Florida Atlantic University, \\ Boca Raton, FL 33431 \\ and \\ William J Devenport, \\ Department of Aerospace and Ocean Engineering, \\ Virginia Polytechnic Institute and State University, \\ 215 Randolph Hall, Blacksburg, VA 24061
}

October 1991

Also published as:

S.A.L. Glegg and W.J. Devenport, "The application of experimental data to blade wake interaction noise prediction", Sixth International Symposium on Unsteady Aerodynamics, Aeroacoustics and Aeroelasticity of Turbomachines and Propellers, University of Notre Dame, IN, September 15-19, 1991.

W.J. Devenport and G. Sharma, "Far-field turbulence structure of the tip vortex shed by a single rectangular wing", 8th Symposium on Turbulent Shear Flows, Munich, Germany, September 9-11, 1991.

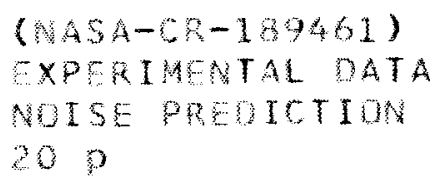




\title{
The Application of Experimental Data to Blade Wake Interaction Noise Prediction
}

\author{
S.tewart Glegg ${ }^{1}$ \\ William J. Devenport ${ }^{2}$
}

\begin{abstract}
Blade Wake Interaction noise (BWI) was defined by Brooks et al [3] as the broadband noise generated by the ingestion of turbulent trailing tip vortices by helicopter rotors. This was shown to be the dominant contributor to the subjectively important part of the acoustic spectrum for the approach stage of a helicopter flyover. Glegg [6] developed a prediction method for BWI noise based on the calculated trailing vortex trajectories and estimates of the vortex turbulence based on measurements by Phillips and Graham [11]. These measurements were made on a trailing vortex from a split wing arrangement and did not give the spectrum of the velocity fluctuations. This paper will describe a recent experiment which has been carried out to measure the turbulence associated with a trailing vortex and the application of the results to BIII noise prediction.
\end{abstract}

\subsection{Introduction}

Experimental measurements, by Brooks et al [3], have identified the midfrequency broadband noise from helicopter rotors to be generated by the interaction of the rotor with its own wake. A typical noise spectrum for a rotor in forward flight is shown in figure 1.1, and this demonstrates that, for constant rotor thrust, the broadband noise level is a strong function of the tip path plane angle. As the tip path plane angle is increased the spectral level drops significantly, and this has been shown by Glegg [0] to correlate with the ingestion of the wake into the forward sector of the rotor disc plane. At small values of the tip path plane angle the rotor wake is close to

\footnotetext{
'Center for Acoustics and Vibration, Department of Ocean Engineering, Florida Atlantic University, Boca Raton, Florida. 33431.

${ }^{2}$ Department of Aerospace and Ocean Engineering, Virginia Polytechnic and State University, Blacksburg, Virginia 2\$061
} 
the blades, but as the tip path plane angle is increased the wake moves away from the rotor and there is a corresponding reduction in noise level. This noise mechanism was defined by Brooks et al [3] as Blade Wake Interaction (BWI) noise, and has been shown to be the dominant contributor to the subjectively important part of the noise spectrum for the approach stage of a helicopter flyover. A method for predicting BWI noise was developed by Glegg [6] using the calculated trailing vortex trajectories from the rotor blades, and estimates of the vortex turbulence based on measurements by Phillips and Graham [11]. It was found that good agreement could be obtained with the spectral level, and the correct scaling with tip path plane angle was obtained, but the spectral shape was not well predicted. The measurements by Phillips and Graham [11] were made on a trailing vortex from a split wing arrangement and did not give the spectrum of the turbulent velocity fluctuations which define the spectral shape of the radiated noise. Consequently the prediction method estimated the noise spectrum by assuming a Von Karman approximation for the turbulence energy spectrum and estimating the integral lengthscales from similar flows. llowever to obtain a better understanding of BWI noise more details are required on the features of the turbulent flow in a vortex. This paper will describe an experiment which has been specifically designed to measure this flow using a single wing in a wind tunnel, and will show how the measurements can be incorporated into the BWI noise prediction scheme. Comparisons of the results with rotor noise measurements will also be given.

\subsection{Experimental Work}

To provide the information about the turbulence structure and velocity spectrum required for BWI noise predictions velocity measurements have been made on the tip vortex shed by a single rectangular NACA 0012 wing.

\subsubsection{Apparatus and INSTRUMENTATION}

The vortex was produced in the Virginia Tech Stability Wind Tunnel. This facility has a test section $1.83 \times 1.83 \times 7.33 \mathrm{~m}$, the long axis being in the flow direction. Flow through the empty test section is closely uniform and has a very low turbulence intensity $(<0.05 \%)$. The wing, $0.20 \mathrm{~m}$ in chord and $1.22 \mathrm{~m}$ in span, was mounted vertically in the center of the test section (see figure 1.2), the root being bolted to a turntable assembly flushed into the upper wall of the wind tunnel. The turntable allowed the wing to be rotated to any angle of attack about its quarter chord location. At zero angle of attack the wing leading edge was $0.33 \mathrm{~m}$ downstream of the test-section entrance. To minimize possible unsteadiness and non-uniformity that might result from natural transition, the boundary layer on the wing was tripped. $0.5 \mathrm{~mm}$-diameter glass beads were glued to the wing in a random pattern 
covcring a strip exter ding from the $20 \% 1040 \%$ chord locations with an average density of 200 beads $/ \mathrm{cm}^{2}$. A few measurements were also performed with no trip. Helium ')ubble visualizations performed prior to the velocity measurements (Dever.port and Sharma [4], [5]) showed the vortex generated by this wing $t o$ be insensitive to probe interference and relatively stable over a broad raige of conditions. Thirty chordlengths downstream of the wing some very low frequency lateral motions were observed but these had a magnitude of less than $3 \%$ of the wing chord.

Velocities were me:sured using single, cross and triple hot-wire probes mounted $0.84 \mathrm{~m}$ upstream of a traverse gear fuxed in the test section (see figure 1.2). All senscrs were operaled using Dantec 58C01 and 56C17 anemometer and bridge units interfaced through an analogic HSDAS-12 $A / D$ converter to an. IBM AT compatible computer. The HSDAS-12 can sample 4 channels sinultaneously at rates of up to $100 \mathrm{kHz}$ per channel. Raw data was linear zed and processed on line using an 18.8 laboratories PL1250 array prccessor and also stored on optical disc cartridges. All probes were calibratsil before and afler each traverse. Corrections for temperature drift (typically 1 to $2^{\circ} \mathrm{C}$ per hour) were applied according to the method of Bearman.

The single hot-wir:: probe (TSI type 1210T1.5) was used to measurc profiles of mean velocity and turbulence intensity and spectra in the wing boundary layer as it left the trailing edge. The cross and triple hot-wire probes (Dantec types 55P51 and 55P91) were used to measure radial profiles of all mean veloc:ty, Reynolds stress and triple product components in the vorlex at 20,25 as d 30 chordlengths downstream of the wing. Auto and cross spectra of all velocity components were also measured at representative radial positions. To obtain these data with the cross hot-wire probe it was necessary to r.state it bout its axis in steps of 45 degrees. These rotations were accorn] lished using a computer-controlled stepper motor located at the downstream end of the steel tube, connected to the probe stem by a shaft, sprockets and a timing belt.

The axial and pitct sensitivity of all sensors of the cross and triple- wire probes were measures and taken into account during the data reduction, see Sharma [9]. For cross and triple-wire statistical measurements, between 30000 and 90000 vel seity sumples (depending on local turbulence level) were taken at each p:obe position over a total sampling lime of about 50 seconds. For spectra: measurements, data were collected in 50, 10240-point records at a $30 \mathrm{kHz}$ sampling rate. At selected locations 10240-point records were also measured a: a $1 \mathrm{kHz}$ sampling rate.

\subsubsection{RESULTS}

Figure 1.2 shows the co-ordinale directions $(x, y, z)(U, V, W)$ to be used in presenting results. The origin of the streamwise coordinate ' $x$ ' is the lcading edge of the wing tip st zero angle of allack. Coordinates ' $y$ ' and ' $z$ ' are mea- 
sured from the vortex center. Distances are normalized on the wing chord ' $c$ ' and velocitics on the nominal free-stream speed $U_{\text {ref }}$ measured using a pitot-static probe located in the forward part of the test section. Velocity measurements were performed at Reynolds numbers $\left(R_{e c}=U_{r e f}(c / \nu)\right)$ from 130000 to 530000 , angles of attack from $0^{\circ}$ to $7.5^{\circ}$ and streamwise positions $(x / c)$ from 20 to 30 . Because of the volume of results, only a representative sample will be presented and discussed here. Figures 1.3 through 1.7 show velocities and spectra measured with the cross-wire probe during a $z$-wise traverse through the center of the vortex at $x / c=30, R_{e c}$ $=100000$, with the wing at $5^{\circ}$ angle of attack (rotating from the $x$ to the $z$ axis) and the trip attached. This set of conditions will be refered to as the baseline case. In this case the boundary-layers leaving both sides of the wing were fully turbulent. The thicknesses of the suction and pressure-side boundary layers at the trailing edge were $0.052 \mathrm{c}$ and $0.037 \mathrm{c}$ respectively.

The mean-velocity field of the vortex (figure 1.3) is, at first sight, much as would be expected. Tangential velocities, visible in the $V$ component profile, increase to a peak of $0.25 \mathrm{U}_{\mathrm{ref}}$ at the edge of the vortex core, which appears to have a diameter of about $0.09 \mathrm{c}$. The small core size is circumstantial evidence for the relative stability of the vortex and its insensitivity to probe interference. The $U$ profile shows an axial velocity deficit of about $0.12 U_{\text {res }}$ here. Close to the vortex center the normal stress profiles (figure 1.4) are dominated by strong central peaks generated by the small lateral motions of the vortex and the steep $V$ gradient in the core. The flow behavior here is perhaps best illustrated by figure 1.6 , which shows autospectra of the velocity components at the center of the core. In this plot the frequency has been normalized as $\mathrm{fc}_{\mathrm{C}} \mathrm{U}_{\mathrm{res}}$, and the area under each curve is unity. The sharp peaks in these spectra at very high frequencies, $\mathrm{fc} / \mathrm{U}_{\text {res }}>50$ are a consequence of electrical noise. Ignoring these there appear to be three distinct regions in the spectra. The first is located around a peak centered at $\mathrm{fc} / \mathrm{U}_{\mathrm{ref}}=5$. It seems likely that this peak is a result of laminar instability in the vortex core of the type previously observed by Singh and Uberoi [10]. Their velocity measurements made in the core of a vortex from a laminar flow wing, show a regular instability at a frequency of $\mathrm{f} / \mathrm{U}_{\text {ref }}=3.5$. We suspect laminar flow in the core of the present vortex because comparisons of spectra measured inside and outside the core show an order of magnitude less energy in the former at frequencies typical of small scale turbulence. The second region, located at very low frequencies ( $\mathrm{fc} / \mathrm{U}_{\text {ref }}<$ about 0.3 ) is a direct consequence of the low-frequency lateral motions observed in flow visualizations. The energy associated with these motions is surprisingly large and the frequencies surprisingly low. Figure 1.6 shows the peak frequency to be at most $f c / U_{\text {ref }}=0.003$, corresponding to a streamwise lengthscale of at least 300 chordlengths. Cross spectra (not shown here) indicate substantial coherence between $\mathrm{V}$ and $\mathrm{W}$ in this frequency range implying that these long wavelength disturbances have a preferred direction. Given this, the only satisfactory explanation of 
the lateral motions would seem to be that they are a result of low frequency changes in flow direction through the test section. Changes of only $\pm 0.16^{\circ}$ would be sufficient to account for these motions. The third region lies in the approximate frequency range $\mathrm{fc} / \mathrm{U}_{\text {res }}=0.3$ to 1 , where the spectra show a strong inflection. Here the implied lengthscales, between 1 and 3 chords, seem too small to be a result of wind tunnel disturbances and there is little or no coherence between $V$ and $W$ fuctuations, and so the motion is isotropic. We suspect that velocity fuctuations here may be a result of random lateral motions of the core generated as it is buffeted by the surrounding wake turbulence. In turbulent boundary layers 'inactive' motion of this type contributes as much as $30 \%$ to the normal stresses in the near wall region (see Bradshaw [2]). Without the constraint of a solid interface we might reasonably expect such motion to have a much greater effect.

Figure 1.4 shows a first attempt at reducing the variation of the normal stresses in the core in the absence of wind tunnel disturbances. The time records used to calculate these stresses were high-pass filtered at a frequency $\mathrm{fc} / \mathrm{U}_{\text {res }}$ of 0.013 . They thus exclude a large portion of the energy presumeed to result from wind tunnel effects. Outside the vortex core the effects of vortex motions are much smaller and the true turbulence structure is immediately visible in the normal stress profiles (figure 1.5). This structure, however, is somewhat different from that we had expected. Here the measurements show two, if not three, distinct half turns of the wing wake as it spirals around the vortex. These not only appear as peaks in the normal-stress profiles (figures 1.4 and 1.5 ) but, on close inspection, as inflections and depressions the $W$ and $U$ profiles (figures 1.3 ) respectively, centered at $z / c=+0.66,-0.31$ and +0.19 . In contrast to the results arid discussion of previous workers (in particular Phillips and Graham [11]) there appears to be little or no region surrounding the core where successive turns of the wing wake have merged to form a continuous axisymmetric structure.

To illustrate this point figure 1.7 shows a cross section through the vortex at $x / c=30$. This figure contains contours of $u^{2} / U_{\text {res }}^{2}$ drawn by interpolating $y$ and $z$ profiles measured with the triple-wire probe along a spiral path deduced from the positions of peaks in the profiles. Note that contours have not been drawn in the region $y / c>0, z / c>0.5$ since this would have involved extrapolation of the data. Apart from the lack of axisymmetry, the most striking feature of figure 1.6 is the continuous and rapid reduction in $\mathrm{u}^{2}$ with distance along the spiral wake towards the vortex center. (This is despite the fact that turbulence levels in the wake are already very low.) A similar reduction is also visible in all the other stresses (see figures 1.4 and 1.5 ). This may indicate that the greater circumferential shear experienced by the wing wake towards the vortex center inhibits the development of, or breaks up, large stress-producing turbulent eddies. Figure 1.6 does not show whether the reduction in turbulence stresses continues into the core of the vortex, since the true turbulence levels here are obscured by 
the small lateral motions of the vortex. Measurements and discussion of the other cases listed in table 1 are presented by Sharma [9]. One relevant conclusion from that work is that turbulence levels in the spiral wake are very strongly dependent on the properties of the wing boundary layer, e.g. removing the wing trip lowers normal-stress levels by $60 \%$. This and the above observations lead us to believe that the wing tip vortex merely serves to dissipate and diffuse the turbulence of the wing wake.

\subsection{Modelling of the Turbulence}

The turbulent flow measurements in the vortex have identified two different mechanisms for generating the unsteady velocity fluctuations at a fixed point. Close to the vortex center the flow is dominated by the small lateral motions of the vortex core, and at the edge of the vortex the turbulence in the spiral wake is the most important feature. In this section we will consider both of these flows and develop an analytical model to describe their features.

\subsubsection{The Small Lateral Motions of the Vortex}

To specify the unsteady velocity caused by small lateral motions of the vortex we will consider the vortex center as displaced from the center of the co-ordinate system by $z(t)$ which is a random function of observer time at a fixed streamwise location. $A$ turbulent eddy located at $x$ relative to the origin of the co-ordinate system, is displaced from the center of the vortex by $y$, such that $x=y+z(t)$. The mean velocity field of the vortex is defined as $V(y)$ and the unsteady turbulence component is $w(y, t)$ and are a function of the displacement from the vortex center. The velocity measured by a fixed probe will have a mean component $U(x)$ and an unsteady component $s(x, t)$, given by

$$
\mathrm{U}(\mathrm{x})+\mathrm{s}(\mathrm{x}, t)=\mathrm{V}(x-z(t))+\mathrm{w}(x-z(t), t)
$$

If the displacements of the vortex are small in comparison with the the core radius then we can expand the right hand side of this equation in a Taylor series, to first order, giving

$$
U(x)+s(x, t)=V(x)+w(x, t)-(z(t) \cdot \operatorname{div})(V+w)
$$

This shows that the measured mean component $U(x)$ is, to first order, the same as the mean flow about the vortex. The unsteady component is a combination of the turbulence associated with the vortex and velocity fluctuations induced by the time varying lateral displacements.

We assume that $w$ and $z=(0, \alpha, \beta)$ are uncorrelated so that the mean 
square velocity components $\mathrm{u}^{(2)}=\left\langle\mathrm{s}^{(2)}\right\rangle$ are given by

$$
u^{(2)}=v^{(2)}+\left\langle\left(\alpha \frac{\partial V}{\partial y}+\beta \frac{\partial V}{\partial z}\right)^{2}\right\rangle
$$

If the vortex displacements can be assumed to be isotropic with an rms displacement $\epsilon$, so that

$$
\left\langle\alpha^{2}\right\rangle=\left\langle\beta^{2}\right\rangle=\epsilon^{2}<\alpha \beta>=0
$$

the three components of the normal turbulent stresses $\mathrm{u}^{(2)}=\left(u^{2}, v^{2}, w^{2}\right)$ due to the lateral vortex motion can be defined, on $y=0$, as

$$
\begin{aligned}
& u^{2}=\epsilon^{2}\left(\frac{\partial U}{\partial r}\right)^{2} \\
& v^{2}=\epsilon^{2}\left(\frac{\partial V_{\theta}^{\prime}}{\partial r}\right)^{2} \\
& w^{2}=\epsilon^{2}\left(\frac{V_{\theta}}{r}\right)^{2}
\end{aligned}
$$

where $U$ is the mean axial velocity and $V_{\theta}$ is the mean azimuthal velocity of the vortex. Schlinker and Amiet [12] and Howe [7] define a model of the azimuthal and axial velocity in a vortex as a function of the radial distance from it's center. This model couples a solid body rotation in the viscous core with a $1 / r$ decay in the outer region. The azimuthal velocity is given by:

$$
V_{\theta}(r)=C \frac{v_{0} r_{0}}{r}\left[1-\exp \left(-\alpha\left(r / r_{0}\right)^{2}\right)\right]
$$

where $\alpha=1.25643$ and $C=(1+0.5 / \alpha)=1.398$. The maximum azimuthal velocity is $v_{0}$ and occurs at a radius of $r_{0}$. The circulation is given by $\Gamma_{0}$ $=2(1+\alpha / 2) v_{0} r_{0}$. The axial velocity deficit model is

$$
U(r)=U_{r e f}-U_{D} \exp \left(-\alpha\left(r / r_{0}\right)^{2}\right)
$$

where $U_{D}$ is the axial velocity deficit on the centerline. The experimental results suggest that $v_{o}=0.25 \mathrm{U}_{\text {res }}, r_{o}=0.045 \mathrm{c}$ and $U_{D}=0.12 \mathrm{U}_{\mathrm{res}}$. In general this model gives a good fit to the azimuthal velocity in the viscous core but underpredicts the velocity in the outer region, suggesting that the field is not decaying as rapidly as expected from the simple model given here. The width of the axial velocity deficit is also underpredicted. However, in this analysis we are concerned with the velocity induced by lateral motions of the vortex core which will not be affected by errors in the outer region or in the axial velocity deficit. By using equations 1.8 and 1.9 in equations 1.5 -1.7 we obtain

$$
\begin{gathered}
u^{2}=\epsilon^{2}\left(U_{D} \frac{2 \alpha r}{r_{0}^{2}} \exp \left(-\alpha\left(r / r_{0}\right)^{2}\right)\right)^{2} \\
v^{2}=\epsilon^{2}\left(C \frac{v_{0} r_{0}}{r^{2}}\left[1-\left(1+2 \alpha\left(r / r_{0}\right)^{2}\right) \exp \left(-\alpha\left(r / r_{0}\right)^{2}\right)\right]\right)^{2}
\end{gathered}
$$




$$
w^{2}=\epsilon^{2}\left(C \frac{v_{0} r_{0}}{r^{2}}\left[1-\exp \left(-\alpha\left(r / r_{0}\right)^{2}\right)\right]\right)^{2}
$$

Notice here that as ' $r$ ' becomes small equations 1.11 and 1.12 can be approximated as

$$
w^{2}=v^{2}=\epsilon^{2}\left(C \frac{\alpha v_{0}}{r_{0}}\right)^{2}=3.08\left(\frac{\epsilon v_{0}}{r_{0}}\right)^{2}
$$

In figure 1.4 we see that $v^{2}=w^{2}=0.0064 \mathrm{U}_{\text {res }}^{2}$ and since $v_{o}=0.24 \mathrm{U}_{\text {res }}$ and $r_{0}=0.045 \mathrm{c}$, we can compute $\epsilon$ as $0.0086 \mathrm{c}$.

Figure 1.8 shows the predicted normal turbulent stresses obtained using this model and the agreement with the measurements in Figure 1.4 is excellent. The only noticeable error is in the predicted value of $u^{2}$ close to $z=0$. However the effects of finite probe size and low turbulence levels at this point may cause the predicted dip to be filled in. The turbulence spectrum of the velocity fluctuations can also be obtained from this model. For example if we consider the spectrum of the velocity components in the $z$ direction, then equation 1.7 implies that

$$
G_{w w}(f)=G_{\iota}(f)\left(V_{\theta} / r\right)^{2}
$$

which indicates that the spectra can be reduced to a universal form by the normalization $G_{w w}(f) / w^{2}=G_{c c}(f) / \epsilon^{2}$. An empirical form for the measured velocity spectra can be obtained using the Von Karman interpolation formula, with a correction for viscous dissipation based on Pao's postulate, and is given by

$$
G_{\iota \varsigma}(f)=12.57 \epsilon^{2} \frac{c}{U_{\text {res }}} \frac{\hat{k}_{1}^{2} \exp \left(-\frac{3}{2}\left(\frac{\dot{k}_{1}}{k_{d} L}\right)^{\frac{1}{3}}\right)}{\left(1+\hat{k}_{1}^{2}\right)^{\frac{11}{6}}}
$$

where $\hat{k}_{1}^{2}=\omega L / U_{\text {ref }}$ The experimental measurements imply that for the very low frequency lateral motions of the vortex (which are not isotropic) that $k_{d} L=30$ and $L / c=50$. For the mid frequency range corresponding to $\mathrm{fc} / \mathrm{U}_{\mathrm{ref}}$ greater than 0.3 the lateral motions are isotropic and the spectra can be estimated using $k_{d} L=15$ and $L / c=0.6$ and $c=0.00275 c$.

\subsubsection{The Turbulence in the Wake Spiral}

If it is assumed that the turbulence in the wake spiral is generated by the blade boundary layers, it may be considered as a distorted form of two dimensional wake turbulence. Wygnanski et al (1986) have shown that the turbulence in the wakes of many different types of body can be scaled on the wake width $L_{0}$ which can be defined in terms of the momentum thickness $\theta$ of the boundary layer on the blade as

$$
L_{o}=0.32 \Theta \sqrt{(x / \Theta+380)}
$$


One of the unexpected results of this study was the remarkably good scaling which this type of normalization gave to the turbulent spectrum of the velocity fluctuations in the wake spiral. Figure 1.9 shows the collapse of the $G_{w w}(f)$ spectra when they are normalized as $G_{w w}(f) U_{r e f} / w^{2} L_{o}$ and plotted against $\left\{L_{o} / U_{r e f}\right.$. An empirical fit to these spectra can be obtained based on the Von Karman interpolation formula as

$$
G_{w w}(f)=w^{2} \frac{4 \gamma L_{0}}{3 U_{\text {ref }}} \frac{\left(1+4.66 \bar{k}_{1}^{2}\right)}{\left(1+\hat{k}_{1}^{2}\right)^{\frac{11}{6}}}\left(C \exp \left(-s \hat{k}_{1}^{2}\right)\right)
$$

where $\hat{k}_{1}^{2}=4 \omega \gamma L / 3 U_{\text {ref }}$ and $s=0.9248\left(c / \gamma \kappa R_{c c} \theta\right)$

where $\gamma$ is defined as the ratio of the integral lengthscale of the turbulence to the wake width and is determined from the measurements to be $\gamma=0.2$. The parameter $\kappa$ is proportional to the turbulence intensity and is found to be 0.2 and the constant $C$ ensures integral closure and is 1.36. This normalized spectrum is shown in figure 1.9 , and gives remarkably good agreement with the measurements in this part of the wake.

\subsection{Noise Predictions}

Blade wake interaction noise is gencrated by the unsteady blade loads which result from the passage of the blades through their own wake. To calculate BWI noise we must define the mean properties of the inflow turbulence at each location of the rotor, and use this as an input to an aeroacoustic model, Amiet [1], Glegg [6], to calculate the broadband noise level in the acoustic far field. The turbulence in the wake is assumed to be dominated by the flow near the trailing tip vortices, and so it is first necessary to define the loci of the blade/vortex interactions, Glegg [6], and then to use a model of the turbulence associated with the vortex to calculate the unsteady flow encountered by the blades. The experimental results suggest that there are two dominant features of the unsteady flow which must be considered. The first is the turbulence in the spiral wake, and the second is the unsteady upwash caused by the small lateral displacements of the vortex. In this section we will consider the noise levels generated by each of these mechanisms.

\subsubsection{Noise Generated by the Spiral Wake}

The turbulence in the spiral wake was found to collapse using a Von Karman energy spectrum which gives a simple formulation for the wavenumber spectrum of the turbulence, and was used in the original BWI noise prediction method, Glegg [6]. However, the measurements show that the turbulence intensities and integral lengthscales are smaller than used in the original model. The modification of the prediction method to incorporate 
the measured spectra is thercfore relativcly straightforward, the major difficulty being to estimate the geometry of immersion of the blade in the spiral wake. This will affect both the local turbulence intensity distribution and the spanwise length of the blade which is exposed to turbulent velocity fluctuations, and will be a function of the displacement of the vortex center from the blade. The vortex displacement can be calculated from the wake code, but the functional dependance of the turbulence intensity distribution on this displacement is complex. However it will only affect the intensity of the radiated noise and will have a minor affect on the spectral shape. Consequently a relatively simple model of the blade immersion in the wake is sufficient to give first estimates of the BWI noise spectrum. Figure 1.10 shows the calculations of the radiated noise spectrum using the wake spiral model in comparison with experimental noise measurements given by Brooks et al [3]. It is seen that the levels are of the correct order of magnitude at the high frequencies, but do not predict the low frequencies well. The peak of the broadband noise in the measured spectra occurs at $1500 \mathrm{~Hz}$, while the peak in the predicted spectra occurs at $4500 \mathrm{~Hz}$. This indicates that the turbulence lengthscales in the spiral wake are significantly

shorter than required to predict BWI noise, and so the turbulence in the wake spiral is not the dominant contributor to the acoustic source levels.

\subsubsection{UNSTEADY MOTIONS OF THE Vortex}

The blade vortex interactions which cause BWI noise occur when the vortex core is perpendicular to the blade span, and so if the vortex is stationary there are no velocity fluctuations which occur as the blade passes through the vortex with a constant displacement from the vortex center. However if the vortex has a random lateral displacement from its mean position then there will be unsteady velocity fluctuations on the blade, since the velocity distribution will move relative to the blade surface. The upwash will be anti-symetric about the instantaneous spanwise location of the vortex core, and the spatial location of the distribution will be time varying in blade based co-ordinates. The anti-symetric feature of the upwash gives an anti-symetric unsteady load distribution which is acoustically inefficient, since it is equivalent to a quadrupole source mechanism. Also the lateral motions of the vortex are predominantly of low frequency, and so it would appear that this mechanism of generating unsteady loads on the blades is not acoustically important. However, since the spiral wake turbulence does not account for the measured BVI noise spectrum, calculations have also been carried out to evaluate the noise generated by the lateral motions of the vortex. The acoustic field from a blade moving through a vortex with lateral motions the flow can be modelled as described in section 3.1. The wavenumber spectrum of the velocity fluctuations on the blade are defined using the spectrum of the lateral displacements $G_{c e}(f)$ and the velocity distribution given by equations 1.2 and 1.8. To obtain the wavenumber 
spectrum it is necessary to evaluate the Fourier transform of the upwash velocity as function of span, which is difficult using the distribution given by equation 1.8 unless the viscous core is ignored. However since the mean flow about the blade will displace the vortex core from its ideal trajectory, it is unlikely that the blade will split the core. The dominant feature of the upwash will therefore be the outer region of the vortex which has an azimuthal velocity distribution which is inversely proportional to the the distance from the core. An upwash field which has this velocity distribution can be easily integrated to give the wavenumber spectrum required by the noise prediction scheme. The main difficulty therefore comes in estimating the $G_{e e}(f)$ spectrum, which is currently not well understood, since it could be caused by many different mechanisms. However using the spectrum given by equation 1.14 an estimate of the importance of the lateral motions of the vortex can be obtained.

The analysis shows that the acoustic efficiency will have a quadrupole type directionality, given by $z_{0} y_{0} /\left|x_{0}\right|^{2}$ for an observer at

$$
x_{0}=\left(x_{0}, y_{0}, z_{0}\right)
$$

in blade based co-ordinates, and will scale with frequency as

$$
\omega^{4} \exp \left(-\left|\omega y_{0} \Delta / c_{\circ}\right| x_{o} \mid\right)
$$

where $\Delta$ is the displacement of the vortex from the blade in the $z$ direction, and $c_{o}$ is the speed of sound. This demonstrates that as $\Delta$ increases, there will be a change in the spectral shape of the radiated noise. When $\Delta$ becomes comparable with the acoustic wavelength the exponential term will reduce the noise levels at high frequencies, $(\Delta>>\lambda)$, while not affecting the levels at low frequencies $(\Delta<<\lambda)$. The minimum distance between the vortex core and the blade is therefore an important parameter and it is assumed that this must be at least one core radius. Using the parameters given in section 1.3 for the mid frequency region of the turbulent spectrum and calculating the wavenumber spectrum as described above enables a prediction to be made of the BWI noise caused by this mechanism. This prediction will depend on estimates of the circulation in the vortex core and the radius of the core, which are difficult to estimate for a helicopter rotor which has a continuously varying angle of attack. Using reasonable estimates of these parameters enables a best fit to the measured noise spectrum to be obtained as shown in figure 1.10. The results compare favorably, but the predictions are significantly less than the measured levels. At the present time the scaling of the mid frequency turbulence spectrum on the now parameters is not completely defined, and so applying the results to a helicopter rotor configuration can only give crude estimates of the radiated noise levels. However, based on the approaches described above it appears more likely that the mid frequency turbulence spectrum, which is currently considered to be caused by isotropic lateral motions of the vortex, is the main contributor to the BIVI noise spectrum. 


\subsection{Conclusion}

This paper has described wind tunnel measurements of the unsteady flow about a trailing tip vortex and how they may be applied to the prediction of helicopter BWI noise. The flow measurements have identified three different frequency regions of the flow. A low frequency region caused by variations of the mean flow in the wind tunnel which causes anisotropic lateral motions of the vortex, and induce large unsteady velocities at a point due to high velocity gradients in the vortex core. A mid frequency region in which the vortex is displaced isotropically from its mean value and a high frequency region which occurs in the spiral wake which is wrapped around the vortex core. The turbulence levels in the spiral wake fall as the core is approached and flow in the core itself appears laminar. Estimates of the noise produced by these different frequency regimes of the turbulence show that it is the mid frequency region of isotropic vortex motions which is the most important mechanism for noise production.

Acknowledgments: This work was supported by NASA grant No 1-1119, and the authors would like to thank Dr. T.F. Brooks for useful discussions during the course of the project. The authors thank Barry Steer for his help in preparing this document using $\mathrm{LT}_{\mathrm{E}} \mathrm{X}$.

\subsection{REFERENCES}

[1] Amiet R., 1975, "Acoustic radiation from an airfoil in a turbulent stream", Journal of Sound and Vibration, Vol 41, pp 407-420

[2] Bradshaw P., 1967, "Inactive motion and pressure fluctuations in turbulent boundary layers", J. Fluid Mechanics, Vol 30, Pt 2, pp 241-258

[3] Brooks, T.F., Marcolini, M. A., and Pope, D.S., February 1987. "Main rotor noise study in DNW." Presented at the AlIS Specialist Meeting in Aerodynamics and Aeroacoustics, Arlington, Texas.

[4] Devenport W.J. and Sharma G., 1990, "Flow visualizations of a wingtip vortex in the presence of a probe", VPI\&SU, Report VPI-AOE-177, September.

[5] Devenport W.J. and Sharma G., 1991, "Far-field turbulence structure of the tip vortex shed by a single rectangular wing", 8 th Symposium on Turbulent Shear Flows, Munich, Germany, September $9-11$.

[6] Glegg S.A.L., 1989, "The prediction of blade-wake interaction noise based on a turbulent vortex model", AIAA 89-1134, presented at the 12th Aeroacoustics Conference, San Antonio, Texas. 


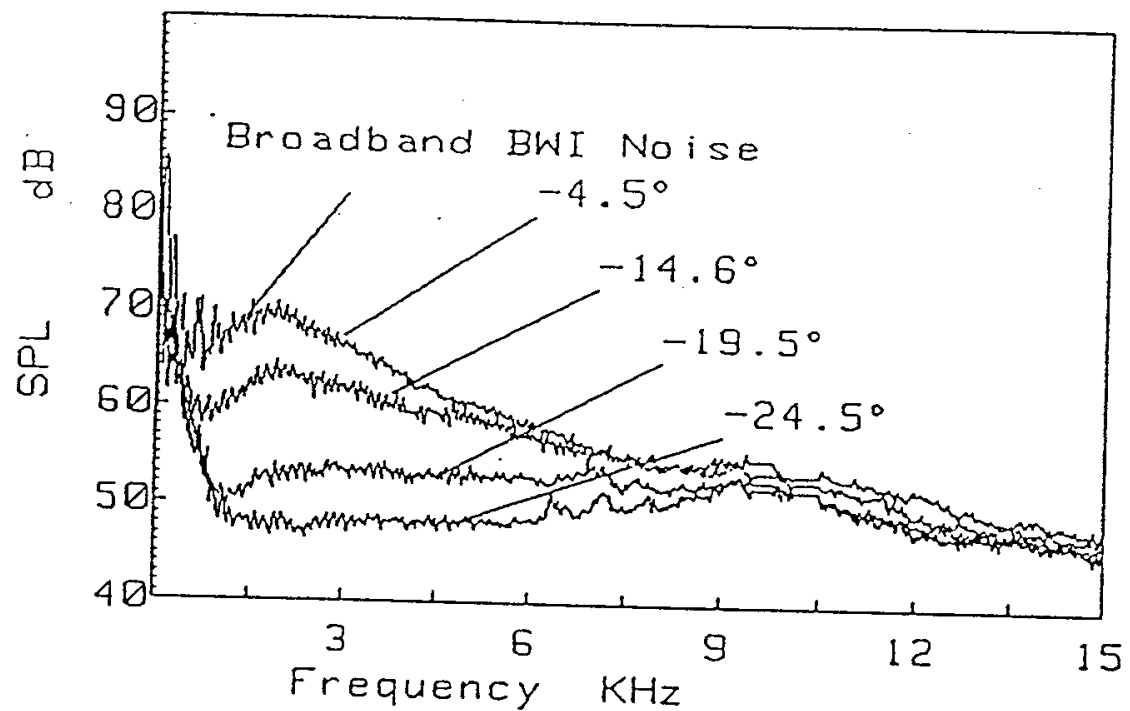

FIGURE 1.1. Measured helicopter noise spectra from a model scale rotor in a wind tunnel showing the broadband BVI noise as a function of the tip path plane angle of the rotor to the Row direction.

[7] Howe M.S., 1988, "Research on Lifting Surface Noise", BBN Report 6890, Bolt Beranek and Newman, Cambridge, Mass.

[8] Mason W H and Marchman J F, 1972, "Farfield structure of an aircraft trailing vortex, including effects of mass injection", NASA CR 62078.

[9] Sharma G., 1991, M.S. thesis, VPI\&SU, expected in September, 1991.

[10] Singh P I and Uberoi M S, 1976, "Experiments on vortex stability", Physics of Fluids, vol 19, p 1858.

[11] Phillips W R C and Graham J A H, 1984, "Reynolds stress measurements in a turbulent trailing vortex" ${ }^{n}$. Journal of Fluid Mechanics, vol 147, p 353.

[12] Schinkler, R. II., and Amiet, R. K., 1983 "Rotor vortex noise" AIAA Paper 83-0720, Presented at the $8 t h$ Aeroacoustics Conference, Atlanta, Georgia.

[13] Wygnanski I., Champagne F.,and Marasli B., 1986, "On the large-scale structures in two-dimensional, small-deficit, turbulent wakes", Journal of Fluid Mechanics vol 168, pp31-il 


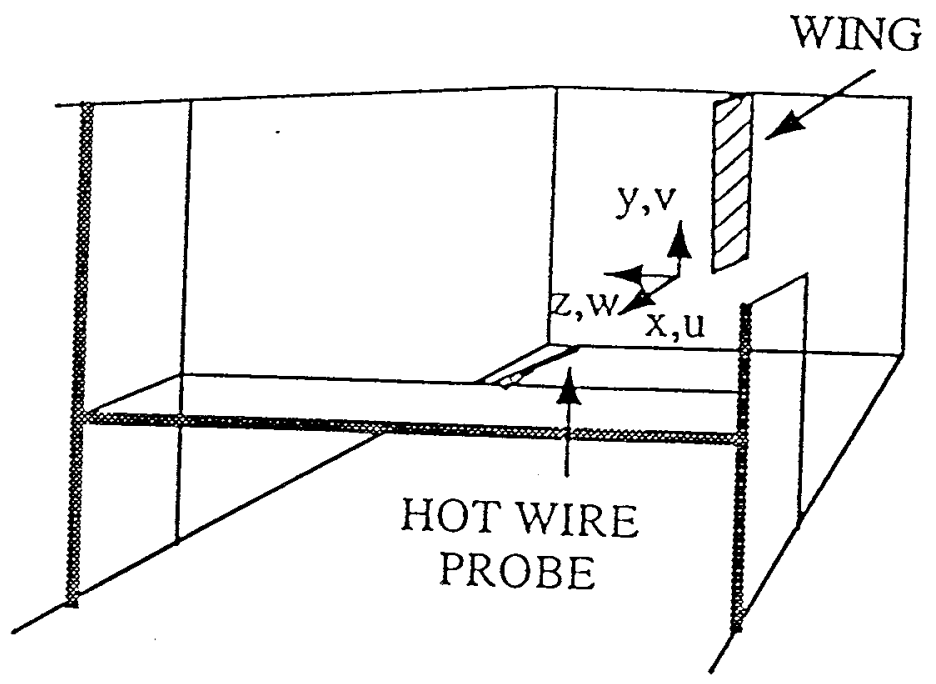

FIGURE 1.2. Schematic of the Stability Wind Tunnel test wing, traverse gear and coordinate system

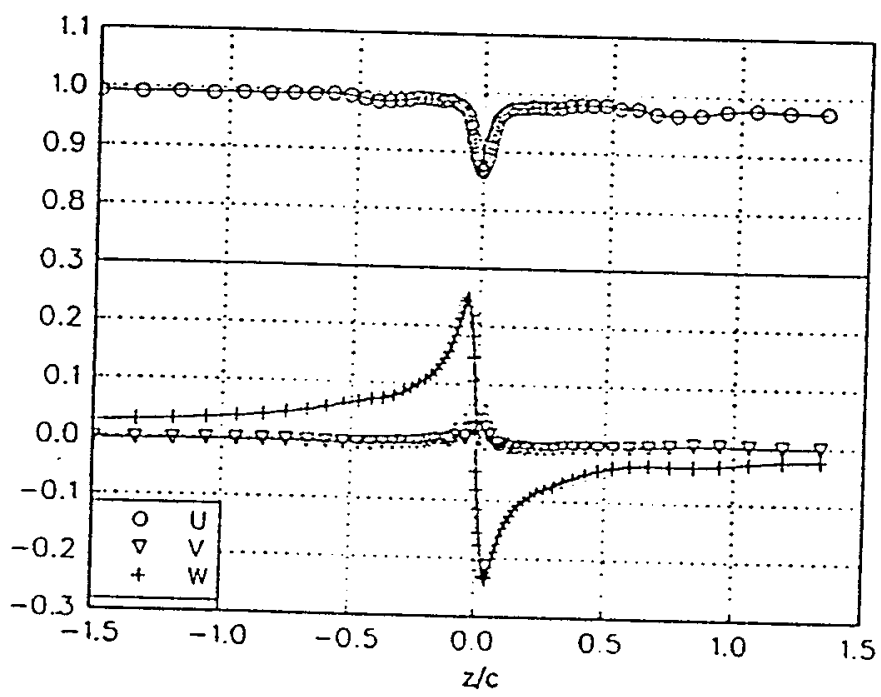

FIGURE 1.3. Velocity profiles measured with the cross-wire probe at $x / c=30$ normalized on $U_{\text {res. }}$ Baseline case. Mean velocities. 


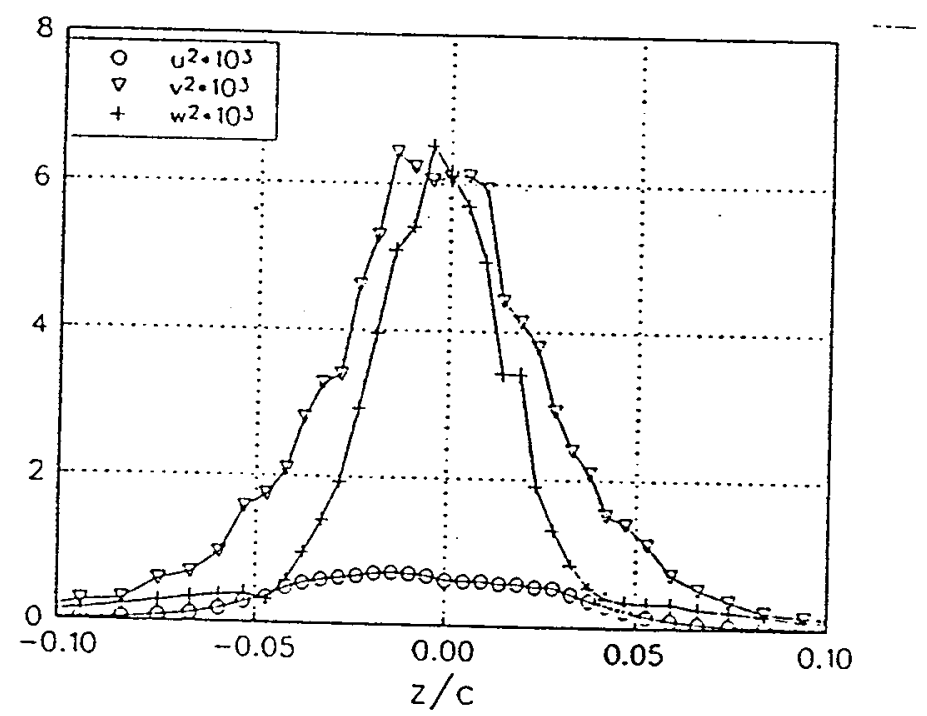

FIGURE 1.4. Velocity profiles measured with the cross-wire probe at $x / c=30$ normalized on $U_{\text {ref. }}$. Baseline case. Filtered normal stress profiles in core.

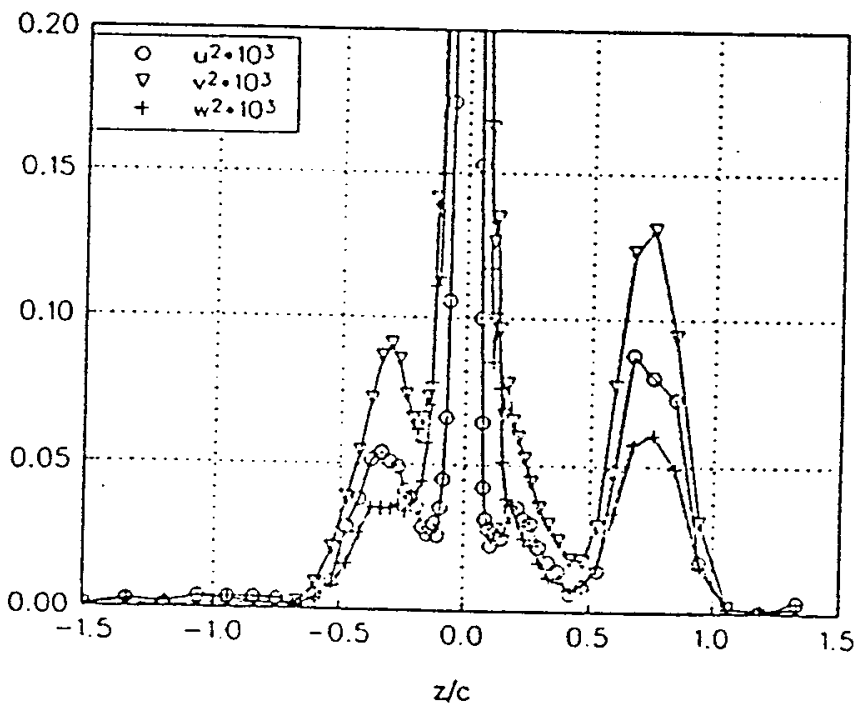

FIGURE 1.5. Velocity profiles measured with the cross-wire probe at $x / c=30$ normalized on $U_{\text {ref. }}$. Baseline case. Normal stress profiles outside core. 


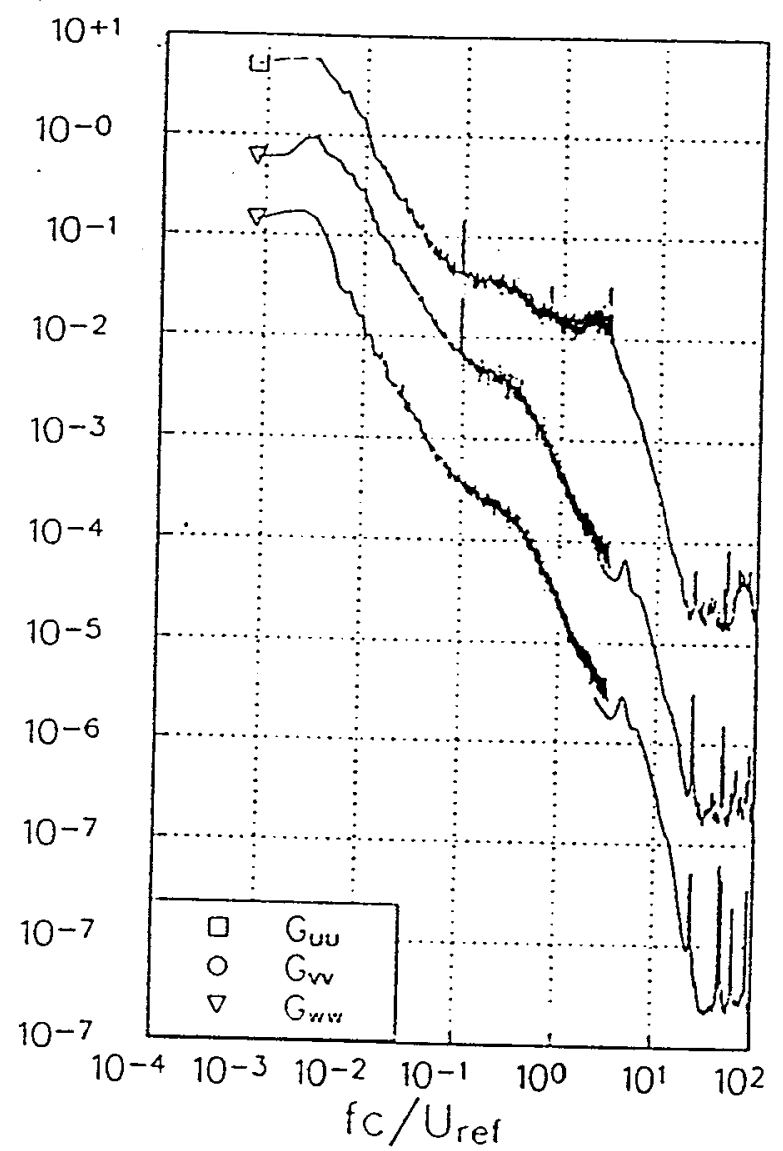

FIGURE 1.6. Autospectra of the normal stresses in the core of the vortex at 30 chords downstream of the blade. 


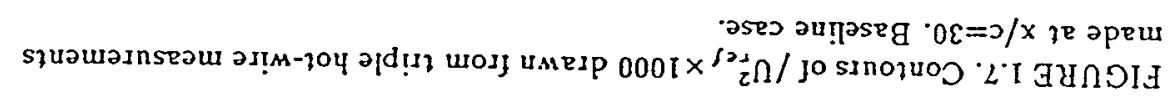

1.

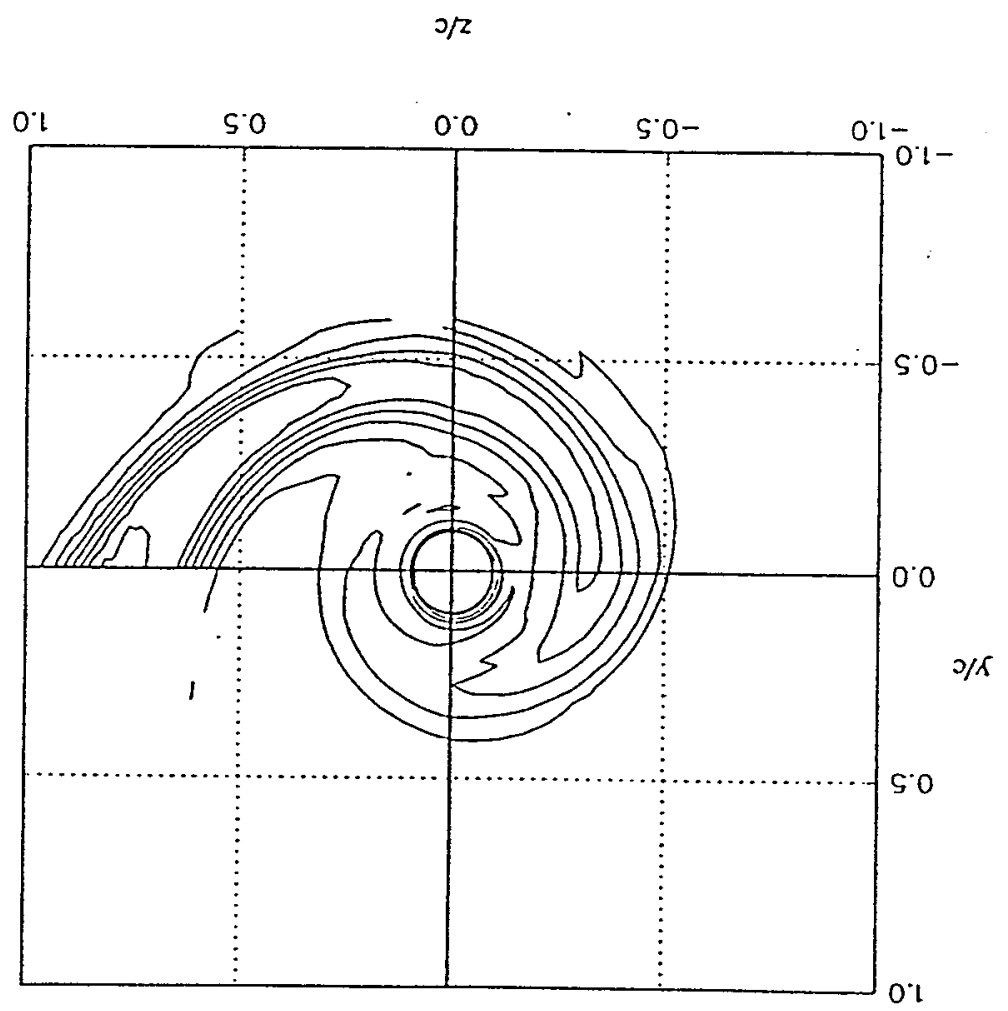




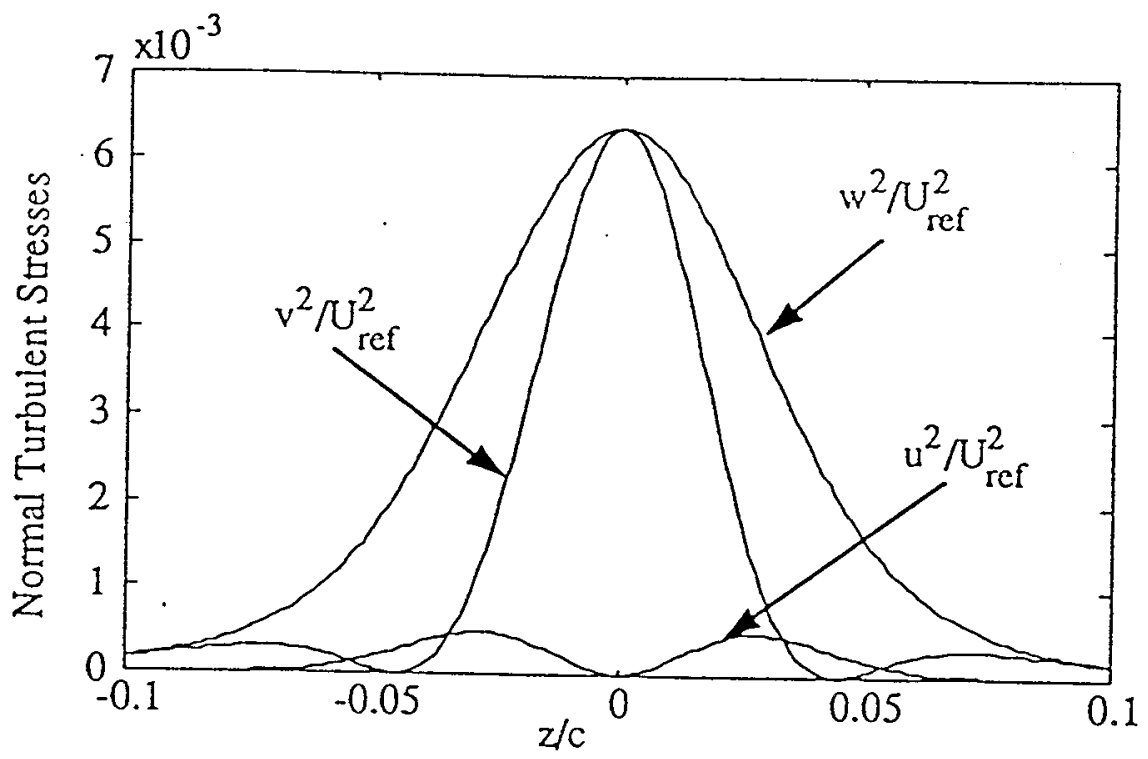

FIGURE 1.8. The predicted normal stresses based on the isotropic lateral vortex motion model.

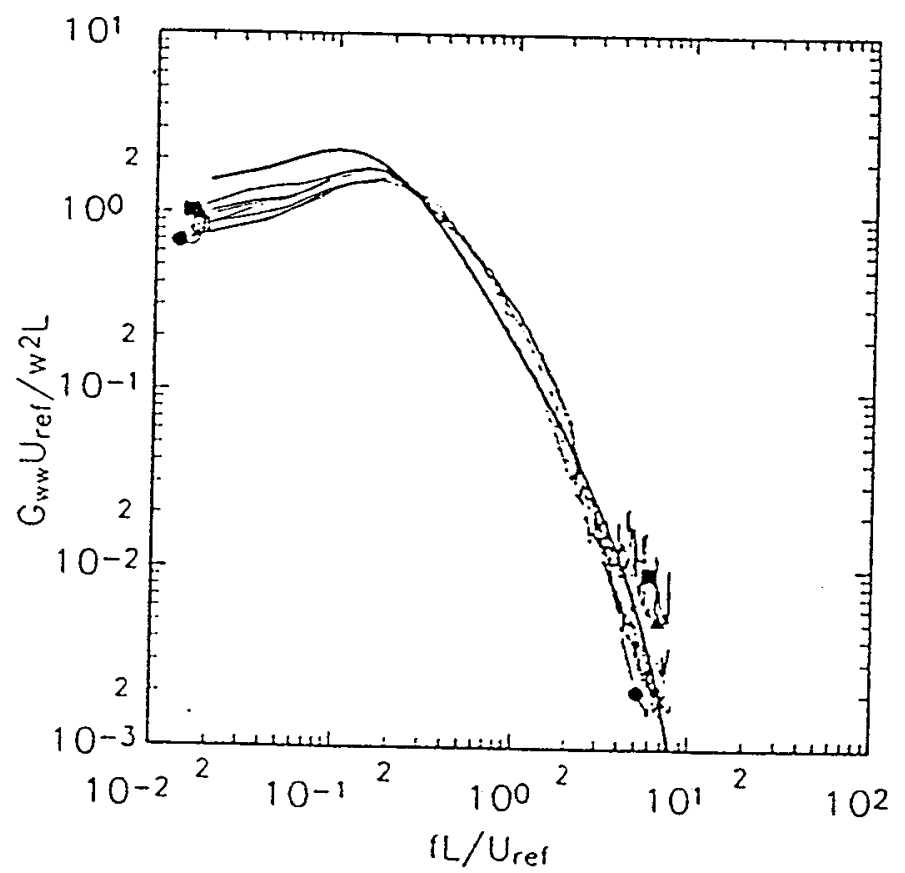

FIGURE 1.9. Autospectra of W-component velocity fluctuations measured in the spiral wake and the estimated spectrum (solid line) using equations $1.10-1.12$. 


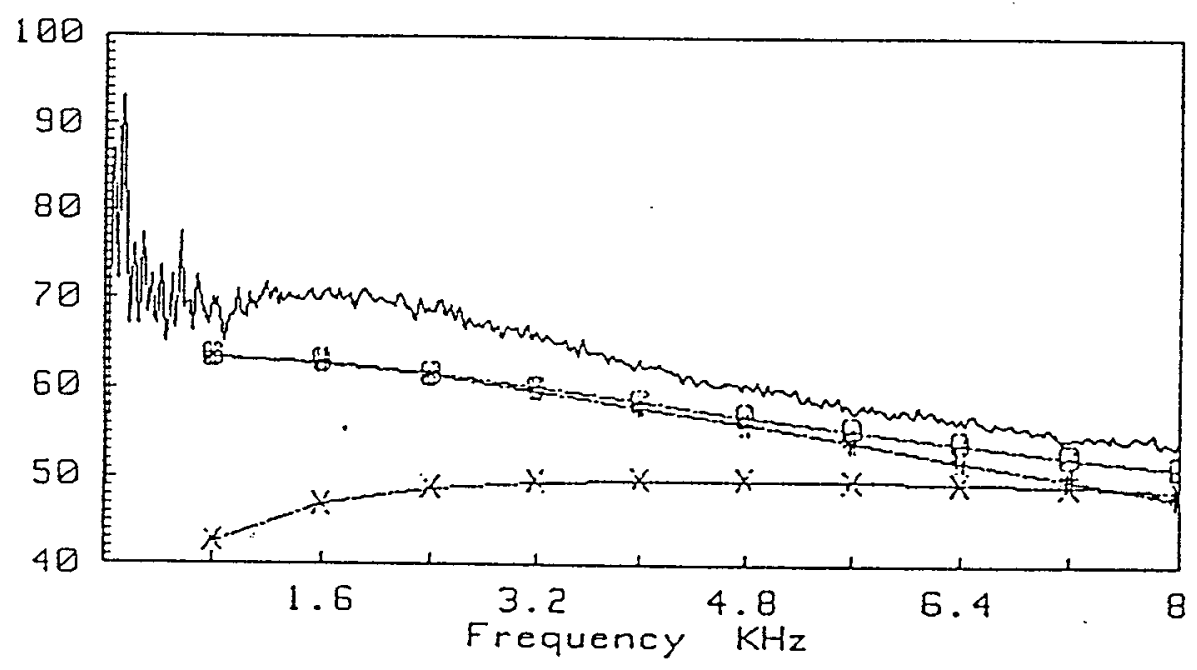

FIGURE 1.10. Comparison between measured and predicted BWI noise levels using the wake model and the lateral vortex motion model. The turbulent wake model lateral vortex motion model total predicted measurements. 